\title{
Surface Effect on Diffractions of Elastic Waves and Stress Concentration near a Cluster of Cylindrical Nanoholes Arranged as Quadrate Shape
}

\author{
Ru Yan \\ Department of Engineering Mechanics, Xian University of Technology, Xian 710048, China \\ Correspondence should be addressed to Ru Yan; ruyan@ymail.com
}

Received 18 September 2014; Accepted 27 November 2014

Academic Editor: Xing Chen

Copyright (C) $2015 \mathrm{Ru}$ Yan. This is an open access article distributed under the Creative Commons Attribution License, which permits unrestricted use, distribution, and reproduction in any medium, provided the original work is properly cited.

\begin{abstract}
We consider the multiple scattering of elastic waves (P-wave and SV-wave) by a cluster of nanosized cylindrical holes arranged as quadrate shape. When the radius of the holes shrinks to nanometers, the surface elasticity theory is adopted in analysis. Using the displacement potential method and wave functions expansion method, we obtain that the multiple scattering fields induced by incident $\mathrm{P}$ - and $\mathrm{SV}$-waves around the holes are derived. The dynamic stress concentration around the holes is calculated to illustrate the effect of surface effects on the multiple scattering of P- and SV-waves.
\end{abstract}

\section{Introduction}

The diffraction of elastic waves by a single inhomogeneity embedded in an elastic medium was discussed in detail by Pao and Mow [1]. With the development of composite materials, there is an increasing demand for understanding the dynamic behavior of composite materials, and much attention has been directed toward the multiple scattering of elastic waves. Fang et al. obtained the multiple diffraction fields by two-particle reinforced composite system [2]. Twersky investigated the scattering of acoustic or electromagnetic wave by an arbitrary configuration of parallel cylinders [3] Lakhtakia et al. [4] observed the reflection and transmission of incident waves by an array of circular cylinders in an elastic slab. Wang and Sudak obtained the scattering field of elastic waves by a cluster of circular cylinders with imperfect interfaces [5].

Nanomaterials have different physical, optical, and mechanical properties distinct from their macroscopic counterparts [6]. At nanoscale, surface has significant effects on the physical and mechanical properties of solids, due to the increasing ratio of surface area to volume $[7,8]$. To account for the surface effects, Gurtin et al. [9] developed a continuum model of surface elasticity. Based on the surface elasticity theory, Wang et al. [10] analyzed the diffraction of plane compressional wave (P-wave) by a nanosized circular hole. $\mathrm{Ru}$ et al. [11] obtained the scattering field of P- and SV-waves by a nanosized inhomogeneity. Wang [12] and Zhang et al. [13] analyze the diffraction of elastic waves by a pair and an array of nanosized inhomogeneities. Recently, Ru et al. discussed the surface effect on the scattering field induced by nanosized cylindrical holes [14]. Those investigations illustrated the importance of surface effects on the diffraction of elastic waves. In this paper, we discussed the multiple scattering of plane P- and SV-waves by a cluster of nanosized cylindrical holes arranged as quadrate shape.

\section{Basic Equations}

At nanoscale, we consider the problem in the framework of surface elasticity theory because of the surface effect [9]. According to the surface elasticity theory, a surface is regarded as a negligibly thin membrane adhered to the bulk without slipping and possesses material constants different from the bulk material. On the surface, the surface stress leads to a set of nonclassical boundary conditions. In the bulk, however, the classical theory of elasticity is still applicable. 


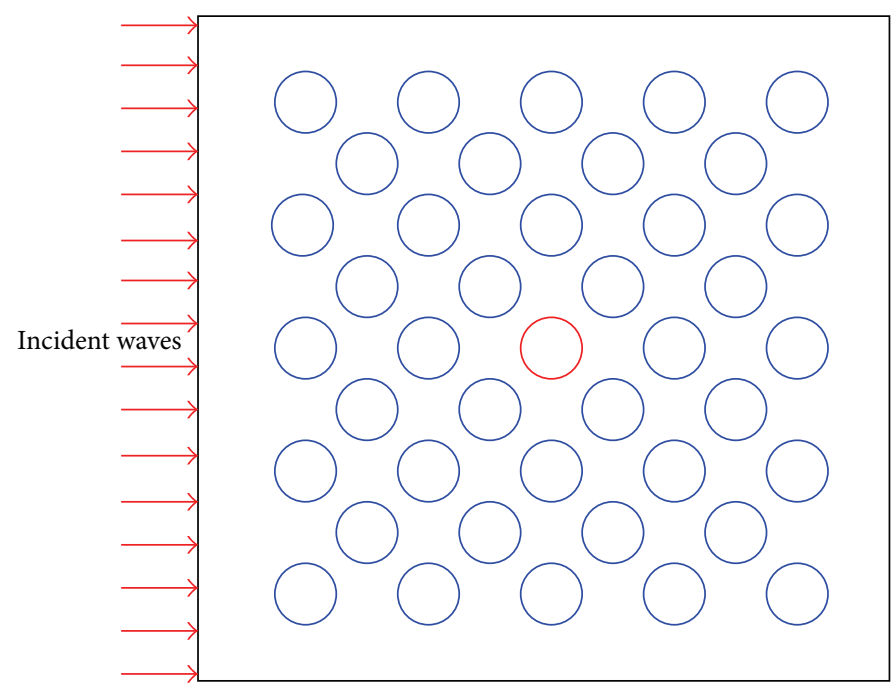

Figure 1: The diffraction of elastic waves by a cluster of infinity nanosized cylindrical holes.

The surface stress tensor $\sigma_{\alpha \beta}^{s}$ is related to the surface energy density $\Gamma$ as [9]

$$
\sigma_{\alpha \beta}^{s}=\Gamma \delta_{\alpha \beta}+\frac{\partial \Gamma}{\partial \varepsilon_{\alpha \beta}}
$$

where $\delta_{\alpha \beta}$ is the Kronecker delta and $\varepsilon_{\alpha \beta}$ is the second-rank tensor of surface strain. In this paper, Einstein's summation convention is adopted for all repeated Latin indices $(1,2,3)$ and Greek indices $(1,2)$.

Without residual surface tension, for an isotropic surface, the relationship between the surface stresses and the surface strains is [9]

$$
\sigma_{\alpha \beta}^{s}=2 \mu^{s} \varepsilon_{\alpha \beta}+\lambda^{s} \varepsilon_{\gamma \gamma} \delta_{\alpha \beta},
$$

where $\mu^{s}$ and $\lambda^{s}$ are two material constants on surface.

Assume that the surface adheres perfectly to the bulk material without slipping, and then the equilibrium equations on the surface are [15]

$$
\begin{gathered}
f_{\alpha}+\sigma_{\beta \alpha, \beta}^{s}=0, \\
\sigma_{i j} n_{i} n_{j}=\sigma_{\alpha \beta}^{s} \kappa_{\alpha \beta},
\end{gathered}
$$

where $f_{\alpha}$ is the tangential component of the traction in the $x_{\alpha}$-direction, $n_{i}$ is the normal vector of the surface, $\kappa_{\alpha \beta}$ is the curvature of the surface, and $\sigma_{i j}$ is stress tensor of the surface. Generally, the surface inertia force can be neglected for dynamic problems.

In the bulk solid, the equilibrium and constitutive equations are the same as those in the classical theory of elasticity:

$$
\begin{gathered}
\sigma_{i j, j}=\rho \frac{\partial^{2} u_{i}}{\partial t^{2}}, \\
\sigma_{i j}=2 \mu\left(\varepsilon_{i j}+\frac{\nu}{1-2 \nu} \varepsilon_{k k} \delta_{i j}\right),
\end{gathered}
$$

where $\rho$ is the mass density of the material, $t$ is the time, $\mu$ and $\nu$ are shear modulus and Poisson's ratio, respectively, and $\sigma_{i j}$ and $\varepsilon_{i j}$ are stress tensor and strain tensor in the bulk material, respectively. by

The strain tensor is related to the displacement vector $u_{i}$

$$
\varepsilon_{i j}=\frac{1}{2}\left(u_{i, j}+u_{j, i}\right)
$$

Based on surface elasticity theory, we derive the solutions for elastic fields near a cluster of cylindrical nanoholes arranged as quadrate shape induced by incident $\mathrm{P}$-wave and SV-wave, respectively.

\section{Diffraction of Elastic Waves by Cylindrical Nanoholes}

We consider the diffraction of elastic waves by a cluster of $N+1$ ( $N$ approaching infinity) identical cylindrical holes with radius of $a$ in an infinite elastic matrix, as shown in Figure 1. The holes are arranged as quadrate shape and the distance between the centers and adjacent holes is $2 b$, as shown in Figure 2. The global polar coordinate system $\left(r_{0}, \theta_{0}\right)$ is set up at the center of the middle hole. For convenience, at the center of $j$ th hole $(j=1,2, \ldots, N)$, the local polar coordinate system $\left(r_{j}, \theta_{j}\right)$ is set up. The plane strain condition $\left(\varepsilon_{z z}=0\right)$ is assumed; thus, $\sigma_{z z}=\nu\left(\sigma_{r r}+\sigma_{\theta \theta}\right)$.

For the present plane strain problem, the surface strain component $\varepsilon_{\theta \theta}^{s}$ on the surface of the hole is given by

$$
\varepsilon_{\theta \theta}^{s}=\frac{1}{2 \mu}\left[(1-\nu) \sigma_{\theta \theta}-\nu \sigma_{r r}\right] .
$$

The surface stress $\sigma_{\theta \theta}^{s}$ can be obtained from (5):

$$
\sigma_{\theta \theta}^{s}=\left(2 \mu^{s}+\lambda^{s}\right) \varepsilon_{\theta \theta}^{s} .
$$




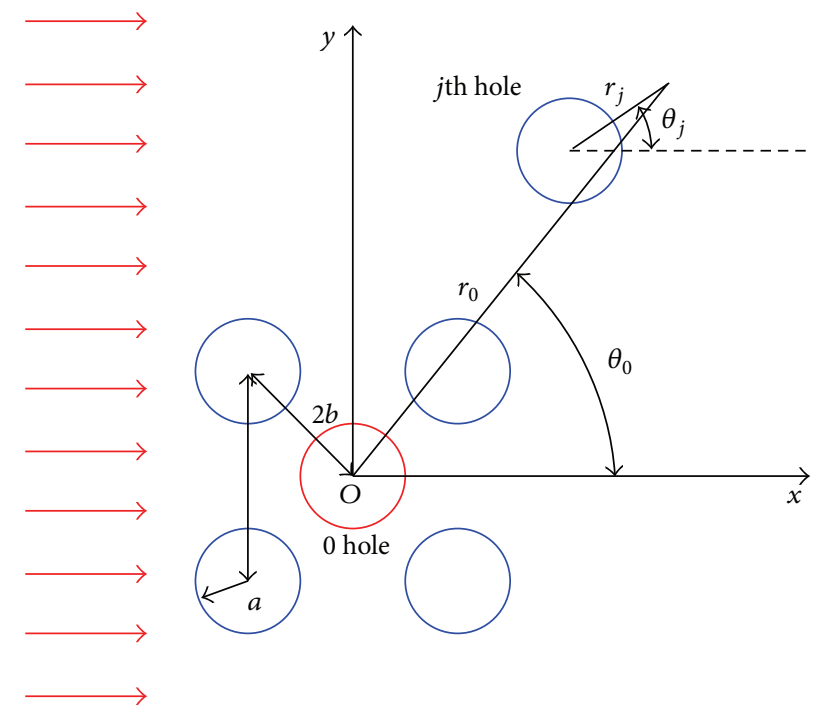

FIgURE 2: Dimensions and coordinate system for formulating the problem.

On the surface, the equilibrium equations ( $3 a)$ and (3b) with surface effects reduce to

$$
\begin{gathered}
\sigma_{r r}=\frac{\sigma_{\theta \theta}^{s}}{a}, \\
\sigma_{r \theta}=-\frac{1}{a} \frac{\partial \sigma_{\theta \theta}^{s}}{\partial \theta} .
\end{gathered}
$$

Substituting (7) into (8) and then into (9a) and (9b), we have

$$
\begin{gathered}
\sigma_{r r}=s\left[(1-\nu) \sigma_{\theta \theta}-\nu \sigma_{r r}\right], \\
\sigma_{r \theta}=-s(1-\nu) \frac{\partial \sigma_{\theta \theta}}{\partial \theta}+s \nu \frac{\partial \sigma_{r r}}{\partial \theta},
\end{gathered}
$$

where

$$
s=\frac{2 \mu^{s}+\lambda^{s}}{2 \mu a}
$$

with $s$ being a dimensionless parameter indicating the effect of surface at nanoscale. Equation (11) shows us that, for a macroscopic inclusion with a big value of $a, s \ll 1$; therefore, the surface effect can be neglected. However, when the radius of the inclusion shrinks to nanoscale, $s$ becomes noticeable and the surface effect should be considered in analysis [1014].

In the bulk solid and the inclusion, the classical theory of elasticity still holds. Therefore, in each of them, the displacements can be expressed by two harmonic potential functions $\varphi$ and $\psi$ (see [1] for more details):

$$
\begin{aligned}
& u_{r}=\frac{\partial \varphi}{\partial r}+\frac{1}{r} \frac{\partial \psi}{\partial \theta}, \\
& u_{\theta}=\frac{1}{r} \frac{\partial \varphi}{\partial \theta}-\frac{\partial \psi}{\partial r} .
\end{aligned}
$$

Using the cylindrical coordinate system, the geometric relations become

$$
\begin{gathered}
\varepsilon_{r r}=\frac{\partial u_{r}}{\partial r}, \\
\varepsilon_{\theta \theta}=\frac{1}{r} \frac{\partial u_{\theta}}{\partial \theta}+\frac{u_{r}}{r}, \\
\varepsilon_{r \theta}=\frac{1}{2}\left(\frac{1}{r} \frac{\partial u_{r}}{\partial \theta}+\frac{\partial u_{\theta}}{\partial r}-\frac{u_{\theta}}{r}\right),
\end{gathered}
$$

and the constitutive relations (5) can be rewritten as

$$
\begin{gathered}
\sigma_{r r}=2 \mu \varepsilon_{r r}+\frac{2 \nu}{1-2 \nu}\left(\varepsilon_{r r}+\varepsilon_{\theta \theta}\right), \\
\sigma_{\theta \theta}=2 \mu \varepsilon_{\theta \theta}+\frac{2 \nu}{1-2 \nu}\left(\varepsilon_{r r}+\varepsilon_{\theta \theta}\right), \\
\sigma_{r \theta}=2 \mu \varepsilon_{r \theta} .
\end{gathered}
$$

Thereby, in terms of the displacement potentials, the stresses can be determined from (12a) to (14c). In what follows, we derive the solutions for the present problems. Since the wave diffractions around every hole are identical, for convenience, we consider only the wave diffractions around the middle hole.

3.1. Diffraction of P-Wave by a Cluster of Cylindrical Nanoholes. Assuming a harmonically plane $\mathrm{P}$-wave propagating in the positive $x$-direction, it can be described by using a displacement potential (see [1] for more details):

$$
\varphi^{(i)}=\varphi_{0} \sum_{n=0}^{\infty} I^{n} J_{n}\left(\alpha r_{0}\right) e^{I n \theta_{0}} e^{-I \omega t}
$$

where $\varphi_{0}$ is the amplitude of the incident wave, $I=\sqrt{-1}, \theta_{0}$ is the angle between $x$-axis and the direction of incident wave, 
$t$ is time, $\omega$ is the circular frequency, and the normalized wave number of P-wave in the bulk is

$$
\alpha=\frac{\omega a}{c_{p}},
$$

with the velocity of P-wave being

$$
c_{p}=\sqrt{2 \mu(1-\nu)(1-2 \nu)^{-1} \rho^{-1}}
$$

For an incident wave, SV-wave and P-wave are reflected from each hole. The displacement potentials of the diffracted waves due to the $j$ th (herein, $j=0,1,2, \ldots, N$ ) cylindrical hole can be written as

$$
\begin{aligned}
& \varphi^{(r)}=\sum_{n=-\infty}^{\infty} A_{n j} H_{n}^{(1)}\left(\alpha r_{j}\right) e^{i n \theta_{j}} e^{-i \omega t}, \\
& \psi^{(r)}=\sum_{n=-\infty}^{\infty} B_{n j} H_{n}^{(1)}\left(\beta r_{j}\right) e^{i n \theta_{j}} e^{-i \omega t},
\end{aligned}
$$

where $H_{n}^{(1)}(x)$ is the $n$th order Hankel function of the first kind, $\beta=\omega a / c_{s}$ is the normalized wave number of SV-wave in the matrix, and $c_{s}=\sqrt{\mu / \rho}$ is the velocity of SV-wave. $A_{n j}$ and $B_{n j}$ are two coefficients to be determined by the boundary conditions.

Moreover, the total waves around the middle hole are determined by [1]

$$
\begin{gathered}
\varphi=\varphi^{(i)}+\sum_{j=0}^{N} \varphi^{(r)}, \\
\psi=\sum_{j=0}^{N} \psi^{(r)} .
\end{gathered}
$$

According to the Graf addition theorem [2], the transformation between two local cylindrical coordinate systems $\left(r_{l}, \theta_{l}\right)$ and $\left(r_{k}, \theta_{k}\right)$ is

$$
\begin{aligned}
& H_{n}^{(1)}\left(\alpha r_{l}\right) e^{i n \theta_{l}} \\
& \quad=\sum_{m=-\infty}^{\infty} H_{n+m}^{(1)}\left(\alpha r_{l, k}\right) J_{m}\left(\alpha r_{k}\right) e^{i(m+n) \theta_{l, k}} e^{i n \pi} e^{-i m \theta_{k}}, \\
& H_{n}^{(1)}\left(\beta r_{l}\right) e^{i n \theta_{l}} \\
& \quad=\sum_{m=-\infty}^{\infty} H_{n+m}^{(1)}\left(\beta r_{l, k}\right) J_{m}\left(\beta r_{k}\right) e^{i(m+n) \theta_{l, k}} e^{i n \pi} e^{-i m \theta_{k}} .
\end{aligned}
$$

Using this theorem, we can get the total waves around the arbitrary hole.

Using Graf addition theorem and then substituting (19a), (19b) and (12a), (12b) into (13a), (13b) and (13c) then into (14a), (14b) and (14c), one obtains the solutions for the stress fields in the bulk. From the surface conditions (10a) and (10b), we can determine the coefficients $A_{n j}$ and $B_{n j}$ in the solutions. Thus, the solutions for the elastic fields induced by incident P-wave near a cluster of cylindrical nanoholes are obtained.
3.2. Diffraction of SV-Wave by a Cylindrical Nanoinclusion. Similarly, we can consider the diffraction of plane SV-wave by a cluster of cylindrical nanoholes arranged as quadrate shape. For an incident plane SV-wave propagating in the positive $x$-direction, it can be described by using a displacement potential [1]:

$$
\psi^{(i)}=\psi_{0} \sum_{n=0}^{\infty} I^{n} J_{n}\left(\beta r_{0}\right) e^{I n \theta_{0}} e^{-I \omega t}
$$

The displacement potentials of the diffracted waves due to the $j$ th (herein, $j=0,1,2, \ldots, N$ ) cylindrical hole can be written as

$$
\begin{aligned}
\varphi^{(r)} & =\sum_{n=-\infty}^{\infty} C_{n j} H_{n}^{(1)}\left(\alpha r_{j}\right) e^{i n \theta_{j}} e^{-i \omega t}, \\
\psi^{(r)} & =\sum_{n=-\infty}^{\infty} D_{n j} H_{n}^{(1)}\left(\beta r_{j}\right) e^{i n \theta_{j}} e^{-i \omega t} .
\end{aligned}
$$

So, the total waves around the middle hole are

$$
\begin{gathered}
\varphi=\sum_{j=0}^{N} \varphi^{(r)}, \\
\psi=\psi^{(i)}+\sum_{j=0}^{N} \psi^{(r) .}
\end{gathered}
$$

Substituting (23a), (23b) and (12a), (12b) into (13a), (13b) and (13c) then into (14a), (14b) and (14c), one obtains the solutions for the stress fields in the bulk and the inclusion, respectively. Again, using the surface conditions (10a) and (10b), the coefficients in the solutions can be determined. Thus, the solutions for the elastic fields induced by incident SV-wave near a cluster of cylindrical nanoholes are obtained. In what follows, we will discuss the surface effect on the elastic-wave-induced stress concentration near the cylindrical nanoholes.

\section{Results and Discussions}

To examine surface effect on the multiple scattering of elastic waves, we consider the dynamics stress concentration around the middle hole.

Determine the dynamic stress concentration factor (DSCF) induced by $\mathrm{P}$-wave as

$$
\operatorname{DSCF}_{p}=\left|\frac{\sigma_{\theta \theta}}{\sigma_{p}}\right|,
$$

and the DSCF induced by SV-wave as

$$
\operatorname{DSCF}_{s}=\left|\frac{\sigma_{\theta \theta}}{\sigma_{s}}\right|,
$$

where $\sigma_{\theta \theta}$ is the hoop stress along the middle hole; $\sigma_{p}=$ $\mu \beta^{2} \varphi_{0}$ and $\sigma_{s}=-(\lambda+2 \mu) \beta^{2} \varphi_{0}$ are the stress intensity in the propagation direction of $\mathrm{P}$-wave and $\mathrm{SV}$-wave, respectively. 


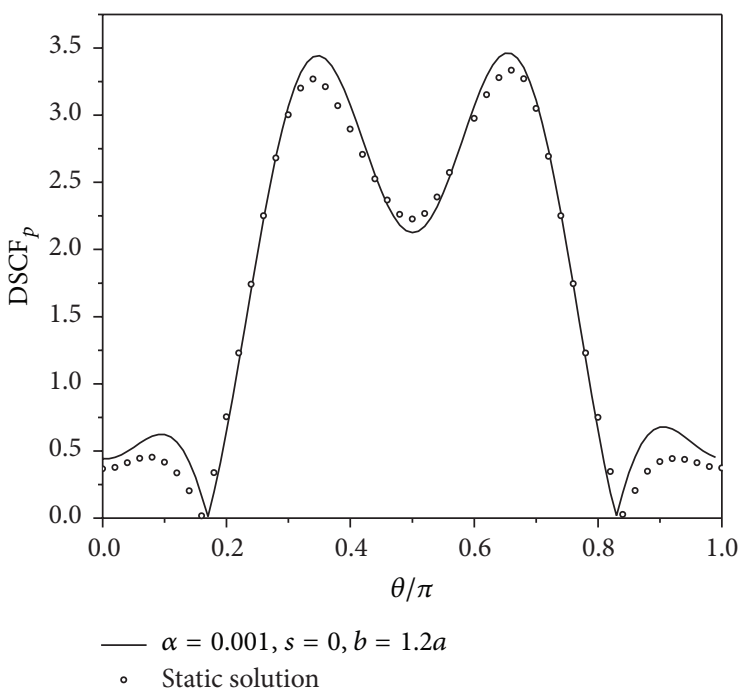

(a)

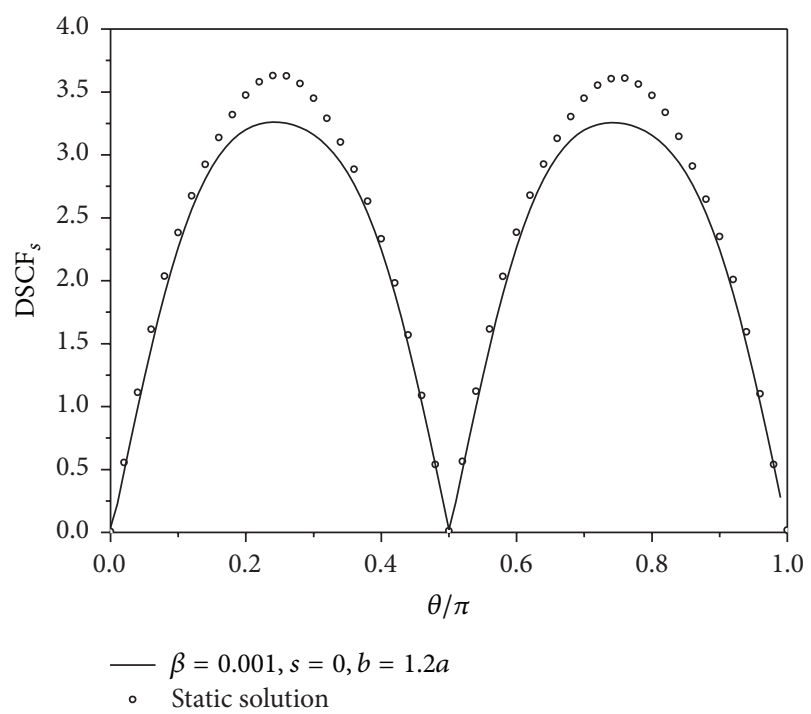

(b)

FIGURE 3: Comparisons of the present numerical results for the DSCF induced by very low frequency incident P-wave and SV-wave with the static solutions.

It is seen that when the surface effect is taken into account, the dynamic stress depends not only on the wave number and Poisson's ratio but also on the surface elasticity parameter $s$ and the distance between holes.

If $\alpha \rightarrow 0$ and keeping $\sigma_{p}$ as a constant, then the incident P-wave represents a static biaxial loading with $\sigma_{x x}=-\sigma_{p}$ and $\sigma_{y y}=-\sigma_{p} \nu /(1-\nu)$ [1]. Similarly, if $\beta \rightarrow 0$ and keeping $\sigma_{s}$ as a constant, then the incident SV-wave represents a static loading with $\sigma_{x y}=\sigma_{s}$ [1]. Moreover, let $s=0$; the present problem reduces to the classical problem without surface effect. For such a remote loading and $b=1.2 a$, we calculate the stress field by ABAQUS. For a low frequency incident wave with $\alpha=10^{-3}$ for P-wave and $s=0$, as shown in Figure 3(a), and for a low frequency incident wave with $\beta=10^{-3}$ for SVwave and $s=0$, as shown in Figure 3(b), the present analytical result agrees well with the static finite element calculation.

For another case, when the distance $2 b$ between holes is much larger than the hole radius $a$, the interaction between holes can be neglected and then the stress field around the hole approaches that for a single hole, as shown in Figures 5, 7,9 , and 11. These two particular cases have been used as a benchmark to test our numerical results.

In what follows, we discuss the effects of the interface at an inclusion on the diffraction of elastic waves and on the dynamic stress concentration factors around the inclusion. We keep $v=0.26$ in the calculations.

\subsection{Dynamic Stress Concentration Induced by P-Wave}

4.1.1. Low Frequency Incident Wave with $\alpha=0.001$. In this case, wavelength $(\lambda=2000 \pi a)$ of the incident wave is much larger than the radius $a$ nanoholes. For $b=1.2 a$, Figure 4 shows the distributions of DSCF around the surface of the middle hole for different values of $s$. The maximum DSCF

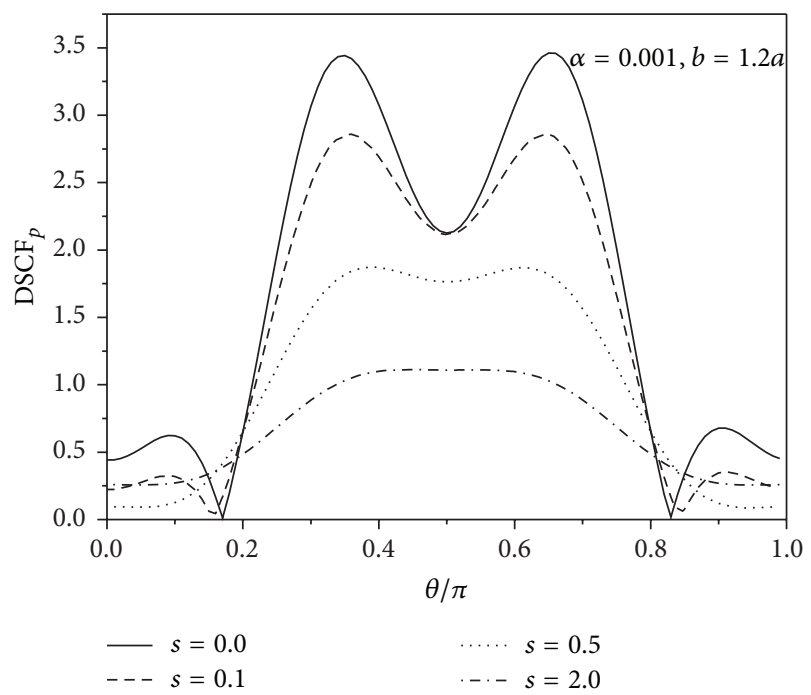

FIGURE 4: Surface effect on the distribution of DSCF along the middle hole for $\alpha=0.001$ and $b=1.2 a$.

appears at about $\theta=0.3 \pi$ and $\theta=0.7 \pi$. For the whole range, DSCF decreases with the increasing of $s$.

For different separation of $b$ and keeping $s=0$, the distributions of DSCF along the middle hole are shown in Figure 5. It is indicated that the larger the separation of $b$ is, the less the interaction between holes is. When the distance between holes is larger than $10 a$, the interaction between holes can be negligible and the distribution of DSCF approaches that of a single hole. When the separation decreases, the DSCF decreases at $0.25 \pi<\theta<0.4 \pi$ and $0.55 \pi<\theta<0.7 \pi$. 


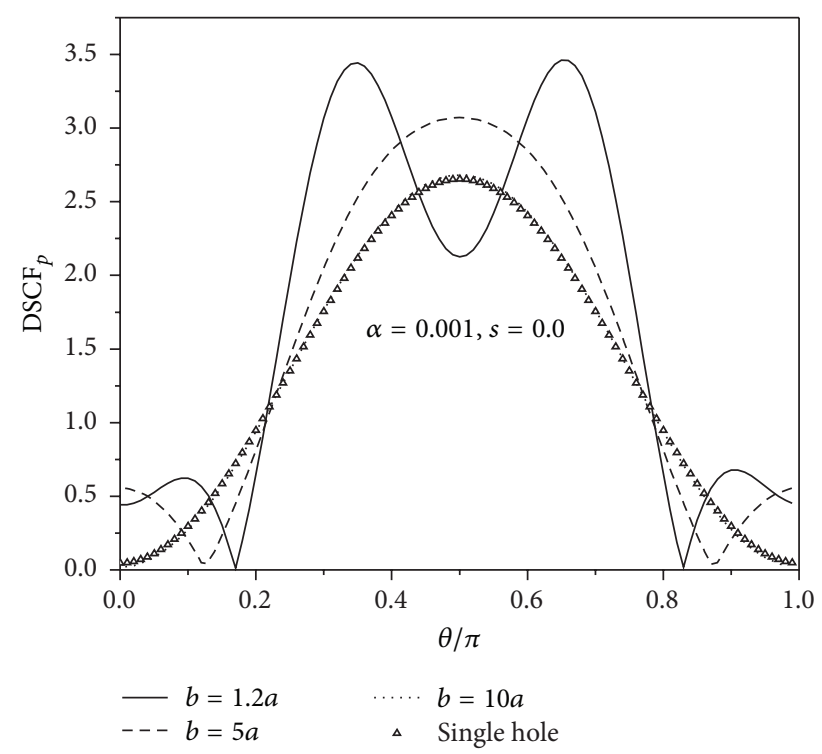

FIGURE 5: Distribution of DSCF along the middle hole for different separation of $b$ for $\alpha=0.001$ and $s=0$.

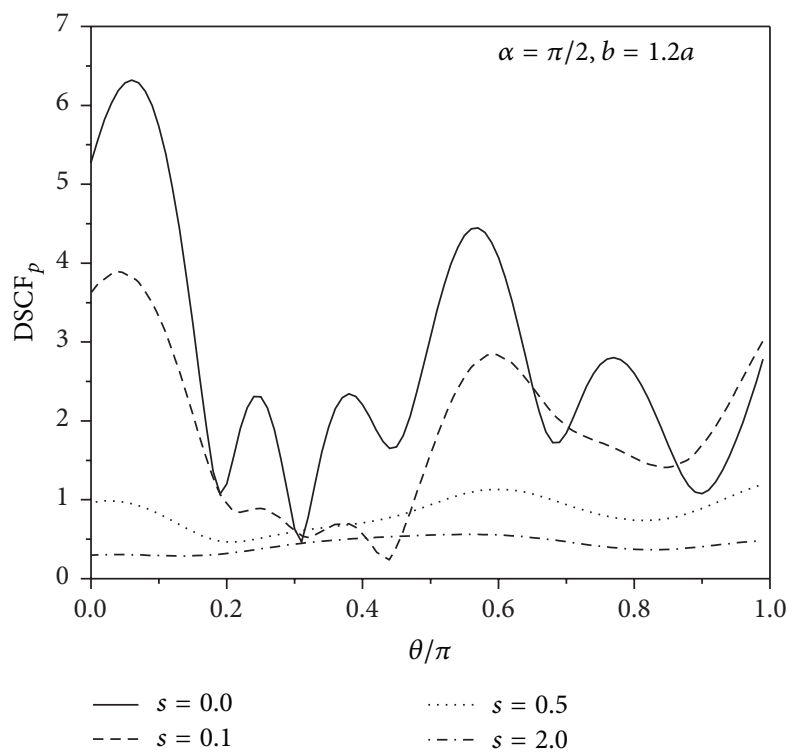

FIGURE 6: Surface effect on the distribution of DSCF along the middle hole for $\alpha=\pi / 2$ and $b=1.2 a$.

4.1.2. High Frequency Incident Wave with $\alpha=\pi / 2$. In this case, the incident wavelength $(\lambda=4 a)$ is comparable with the hole radius. For a small separation of $b=1.2 a$, the distributions of DSCF for various values of $s$ are shown in Figure 6. It is seen that multiple peak values are excited along the surface due to the interference between the incident and reflected waves. With the increasing of $s$, the DSCF decreases almost in the whole range.

Figure 7 shows the variation of DSCF along the hole for different value of $b$. The interference between incident and reflected waves is still obvious. When the separation between holes is large enough (e.g., $b>20 a$ which is much larger than

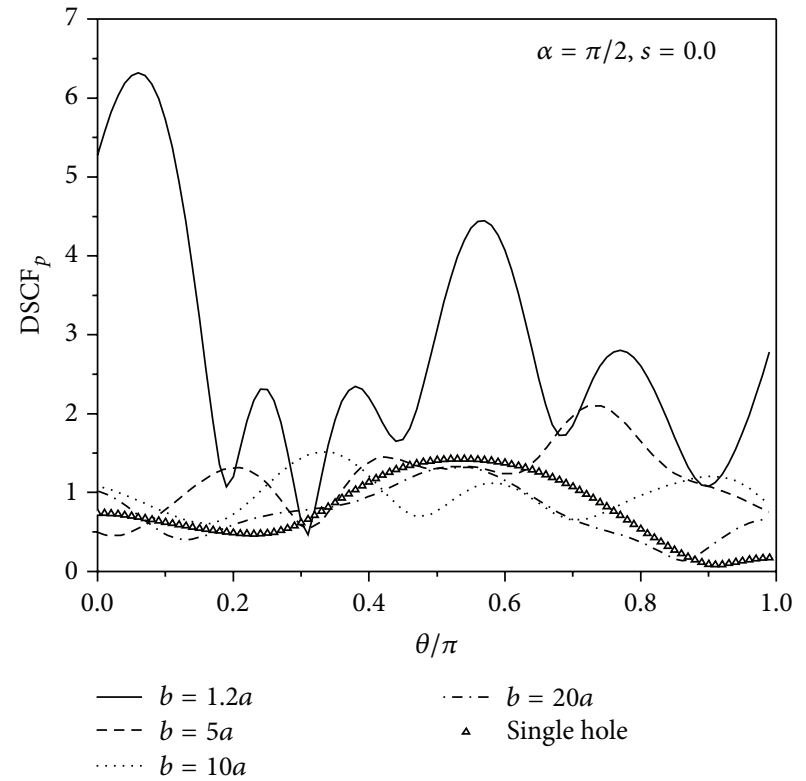

FIGURE 7: Distribution of DSCF along the middle hole for different separation of $b$ for $\alpha=\pi / 2$ and $s=0$.

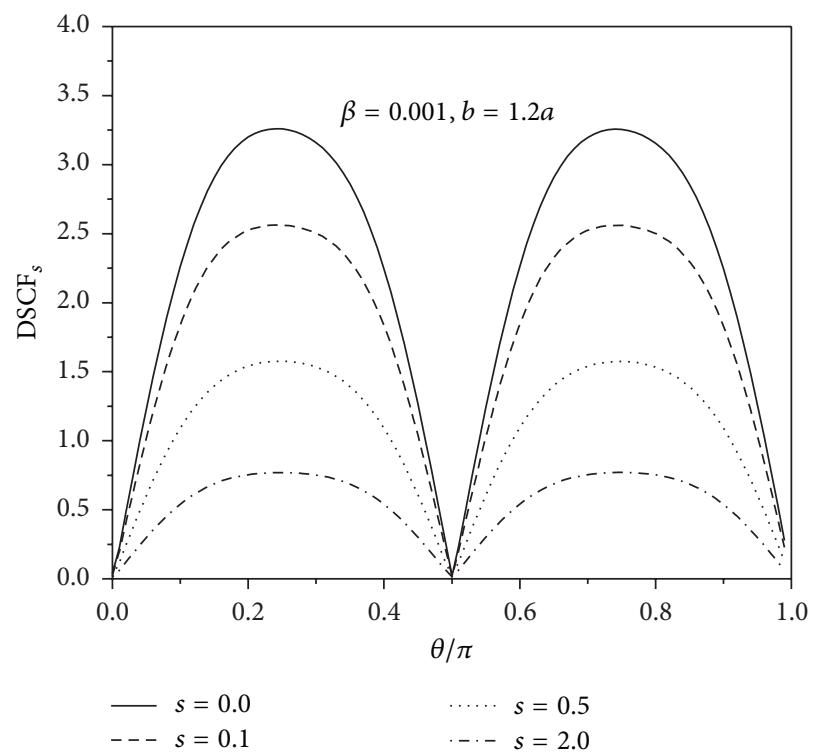

FIGURE 8: Surface effect on the distribution of DSCF along the middle hole for $\beta=0.001$ and $b=1.2 a$.

that for a low frequency), the interaction can be neglected and the distributions of DSCF are approaching to that of a single hole.

\subsection{Dynamic Stress Concentration Induced by SV-Wave}

4.2.1. Low Frequency Incident Wave with $\beta=0.001$. For a small separation of $b=1.2 a$, the distributions of DSCF around the surface of the middle hole for different values of $s$ are indicated in Figure 8. The maximum DSCF appears at almost $\theta=0.25 \pi$ and $\theta=0.75 \pi$. For the whole range, DSCF also decreases with the increasing of $s$. 


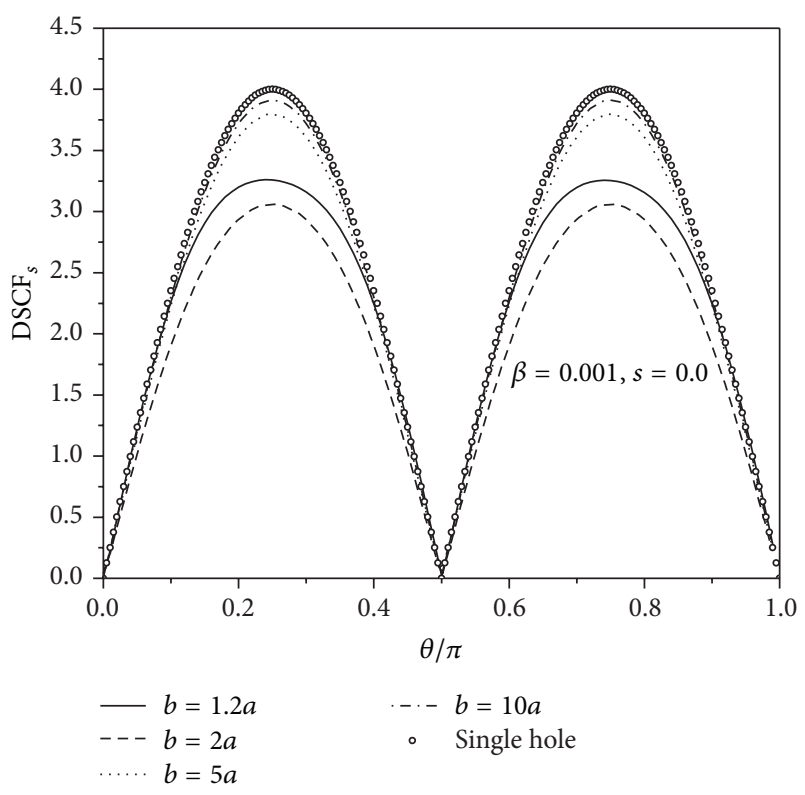

FIGURE 9: Distribution of DSCF along the middle hole for different separation of $b$ for $\beta=0.001$ and $s=0$.

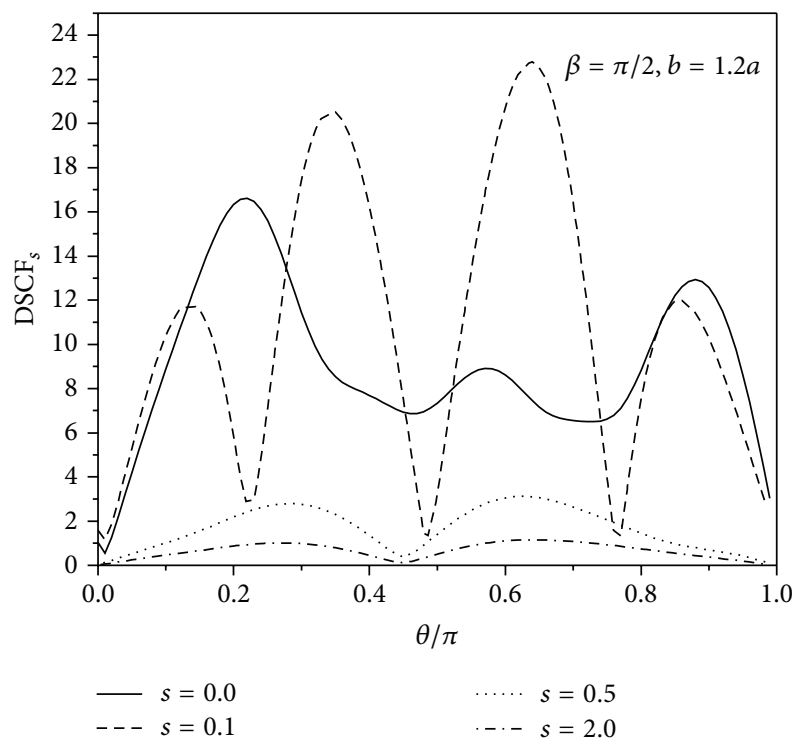

Figure 10: Surface effect on the distribution of DSCF along the middle hole for $\beta=\pi / 2$ and $b=1.2 a$.

For different value of $b$, let $s=0$; the distributions of DSCF along the middle hole are shown in Figure 9. For a larger value of $b$, the interference between holes still holds. When the distance between holes is larger than $10 a$, the distribution of DSCF approaches that of a single hole.

4.2.2. High Frequency Incident Wave with $\beta=\pi / 2$. For $b=$ $1.2 a$, Figure 10 shows the distributions of DSCF for various values of $s$. Multiple peak values are excited along the surface due to the interference between the incident and reflected waves. With the increasing of $s$, the DSCF decreases almost in $0<\theta<0.3 \pi$ and $0.8 \pi<\theta<\pi$.

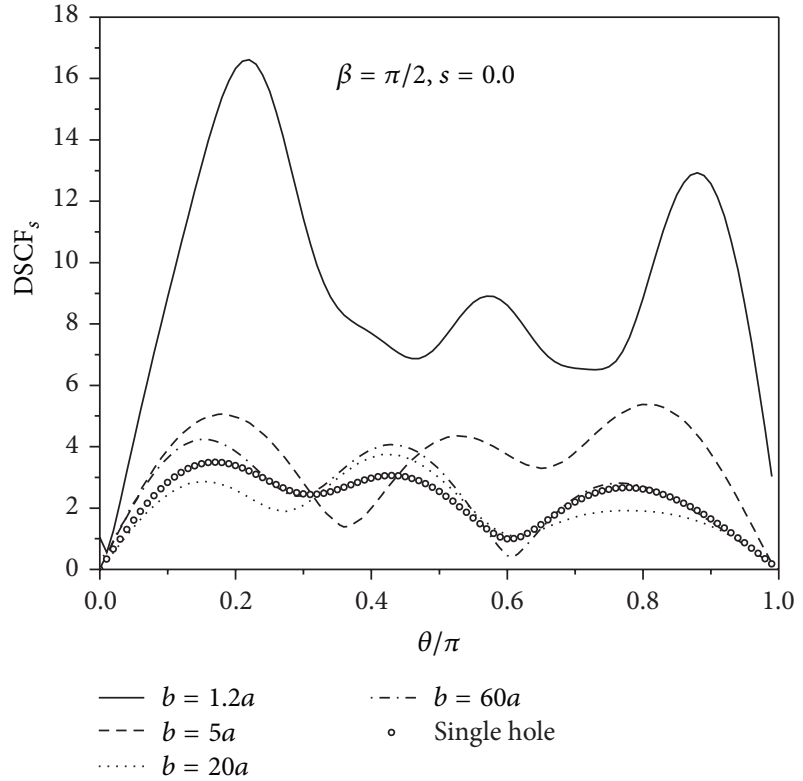

FIGURE 11: Distribution of DSCF along the middle hole for different separation of $b$ for $\beta=\pi / 2$ and $s=0$.

Figure 11 shows the variation of DSCF along the hole for different values of $b$. The interference between incident and reflected waves is obvious. When the separation between holes is large enough $(b>60 a)$, the interaction can be neglected and the distributions of DSCF are approaching to that of a single hole. With the increasing of $b$, the DSCF decreases almost in the whole range.

\section{Conclusion}

Based on the theory of surface elasticity, surface effect on the diffractions of plane elastic waves by a cluster of cylindrical nanoholes arranged as quadrate shape was theoretically investigated in this paper. Solutions for the elastic fields induced by $\mathrm{P}$ - and SV-waves near cylindrical nanoholes are obtained, respectively, and the effects of surface properties on the dynamic stress concentration near the nanoholes are discussed in detail.

It can be concluded that surface effect weakens the phenomenon of dynamic stress concentration. The DSCF depends not only on the surface effects but also on the separation between holes. For both low and high frequency, the interaction between holes is significant in a very small separation. With the increasing of separation between holes, the interaction can be ignored in a small separation for low frequency, but, for high frequency, the interaction can be neglected only for a much large separation.

\section{Conflict of Interests}

The author declares that there is no conflict of interests regarding the publication of this paper. 


\section{Acknowledgment}

This work was supported by the National Natural Science Foundation of China (11302166).

\section{References}

[1] Y. H. Pao and C. C. Mow, Diffractions of Elastic Waves and Dynamic Stress Concentrations, Crane, Russak, New York, NY, USA, 1973.

[2] X.-Q. Fang, C. Hu, and W.-H. Huang, "Scattering of elastic waves and dynamic stress in two-particle reinforced composite system," Mechanics of Materials, vol. 39, no. 6, pp. 538-547, 2007.

[3] V. Twersky, "Multiple scattering of radiation by an arbitrary planar configuration of parallel cylinders and by two parallel cylinders," Journal of Applied Physics, vol. 23, no. 4, pp. 407-414, 1952.

[4] A. Lakhtakia, V. V. Varadan, and V. K. Varadan, "Reflection characteristics of an elastic slab containing a periodic array of circular elastic cylinders: $P$ and $S V$ wave analysis," The Journal of the Acoustical Society of America, vol. 83, no. 4, pp. 1267-1275, 1988.

[5] X. Wang and L. J. Sudak, "Scattering of elastic waves by multiple elastic circular cylinders with imperfect interface," Waves in Random and Complex Media. Propagation, Scattering and Imaging, vol. 17, no. 2, pp. 159-187, 2007.

[6] H. Gleiter, "Nanostructured materials: basic concepts and microstructure," Acta Materialia, vol. 48, no. 1, pp. 1-29, 2000.

[7] E. W. Wong, P. E. Sheehan, and C. M. Lieber, "Nanobeam mechanics: elasticity, strength, and toughness of nanorods and nanotubes," Science, vol. 277, no. 5334, pp. 1971-1975, 1997.

[8] J. H. Davies, "Elastic and piezoelectric fields around a buried quantum dot: a simple picture," Journal of Applied Physics, vol. 84, no. 3, pp. 1358-1365, 1998.

[9] M. E. Gurtin, J. Weissmüller, and F. Larché, "A general theory of curved deformable interfaces in solids at equilibrium," Philosophical Magazine A, vol. 78, no. 5, pp. 1093-1109, 1998.

[10] G. F. Wang, T. J. Wang, and X. Q. Feng, "Surface effects on the diffraction of plane compressional waves by a nanosized circular hole," Applied Physics Letters, vol. 89, no. 23, Article ID 231923, 2006.

[11] Y. Ru, G. F. Wang, and T. J. Wang, "Diffractions of elastic waves and stress concentration near a cylindrical nano-inclusion incorporating surface effect," Journal of Vibration and Acoustics, vol. 131, no. 6, Article ID 061011, 7 pages, 2009.

[12] G. F. Wang, "Multiple diffraction of plane compressional waves by two circular cylindrical holes with surface effects," Journal of Applied Physics, vol. 105, no. 1, Article ID 013507, 2009.

[13] Q. F. Zhang, G. F. Wang, and P. Schiavone, "Diffraction of plane compressional waves by an array of nanosized cylindrical holes," Journal of Applied Mechanics, Transactions ASME, vol. 78, no. 2, Article ID 021003, 2011.

[14] Y. Ru, G. F. Wang, L. C. Su, and T. J. Wang, "Scattering of vertical shear waves by a cluster of nanosized cylindrical holes with surface effect," Acta Mechanica, vol. 224, no. 5, pp. 935-944, 2013.

[15] P. Sharma, S. Ganti, and N. Bhate, "Effect of surfaces on the sizedependent elastic state of nano-inhomogeneities," Applied Physics Letters, vol. 82, pp. 535-537, 2003. 

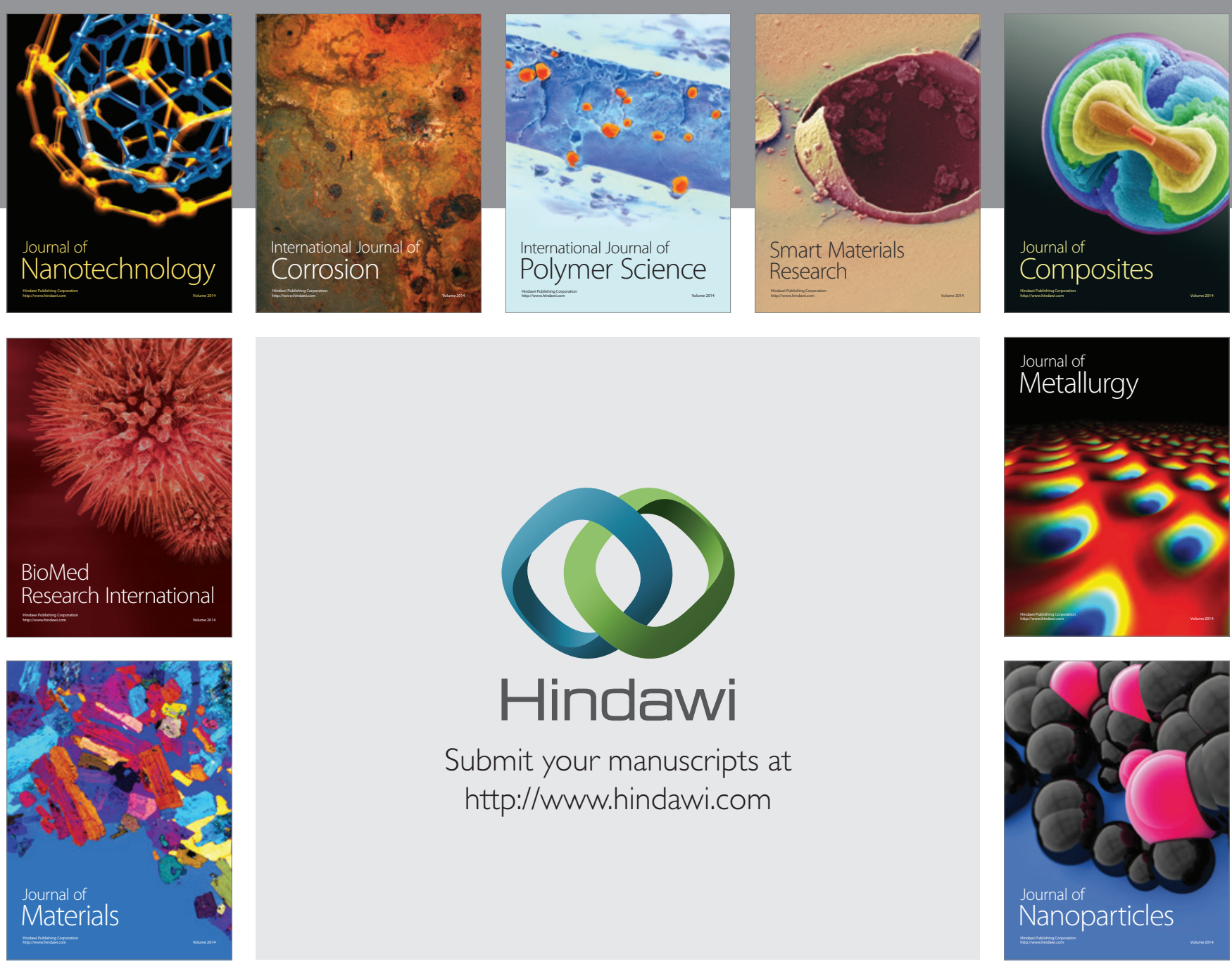

Submit your manuscripts at http://www.hindawi.com
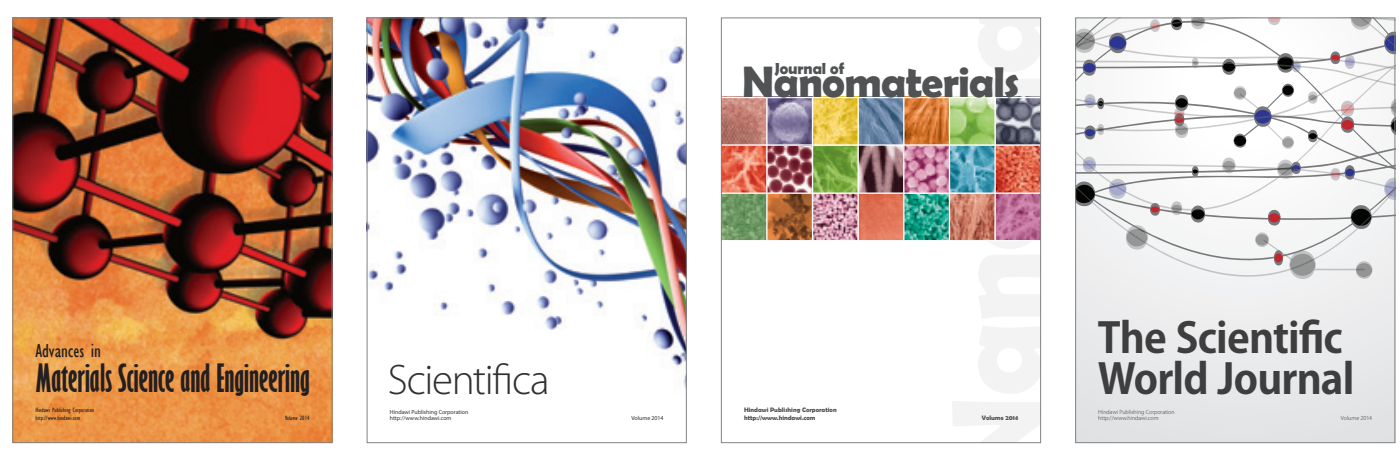

\section{The Scientific World Journal}
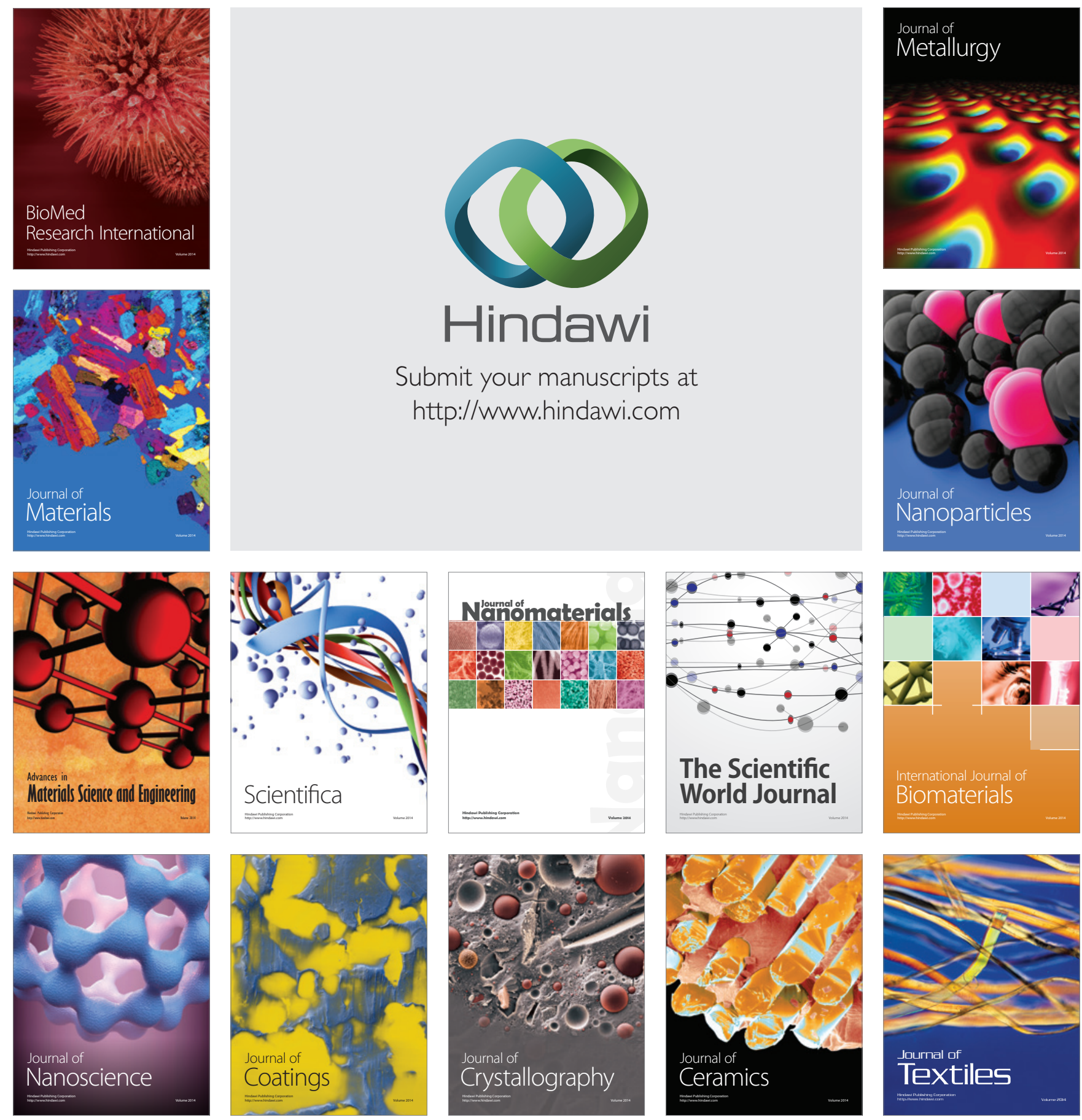